\title{
Splenic Flexure Mobilization for Subacute Large Bowel Obstruction following Gastric Bypass: A Case Report
}

\author{
Tafadzwa P Makarawo ${ }^{1,2^{*}}$, Sarah Bastawrous ${ }^{3}$ and Amir Bastawrous ${ }^{2}$ \\ ${ }^{1}$ Colon and Rectal Surgeon, Colon and Rectal Center of Arizona, USA \\ ${ }^{2}$ Colon and Rectal Surgeon, Swedish Colon and Rectal Clinic, USA \\ ${ }^{3}$ Department of Radiology, University of Washington School of Medicine, Seattle, WA USA; \\ Department of Diagnostic Imaging, VA Puget Sound Health Care System, Seattle, WA USA
}

*Corresponding author: Tafadzwa P Makarawo, Colon and Rectal Surgeon, Colon and Rectal Center of Arizona, USA; Colon and Rectal Surgeon, Swedish Colon and Rectal Clinic, 1101 Madison, Suite 510 Seattle, WA 98104, USA, Tel: +1-206386-6600, Fax: +1-206-386-2452, E-mail: Tafadzwa.Makarawo@Swedish.org

\begin{abstract}
We present the interesting case of a patient with large bowel obstruction following laparoscopic Roux-en-Y gastric bypass surgery.
\end{abstract}

\section{Introduction}

Laparoscopic Roux-en-Y bypass (RYGB) is the approach of choice for bariatric surgical procedures, being performed in the majority of the 200,000 procedures performed in the United States in 2007 [1]. An established complication of laparoscopic gastric bypass surgery is bowel obstructions with a reported incidence as high as 9.7\% [2]. Small bowel obstruction represents the predominant source of obstructions and is typically due to closed loop obstruction within internal hernias [3]. This feared complication can have devastating consequences, such as long segment of small bowel ischemia, if not identified on time [1]. It is therefore a complication that requires a high index of suspicion and a low threshold for early intervention.

Large bowel obstruction, however, has not been reported in association with gastric bypass surgery. The relatively fixed nature and large caliber of the colon limits the risk of involvement with the mesenteric defects that typically trap and incarcerate the small bowel leading to obstruction.
We present the first reported case of a large bowel obstruction secondary to a Roux-en-Y gastric bypass including the surgical method used to correct this unusual complication of bariatric surgery.

\section{Case Report}

This is a case of a 31-year-old Caucasian female that presented with a prior history of multiple bariatric surgeries including laparoscopic gastric band placement in 2006 that was converted to a laparoscopic sleeve gastrectomy in 2010 due to gastric reflux symptoms. Due to severe recurrence of her intractable gastric reflux symptoms despite maximal medical therapy, the patient then underwent a third bariatric procedure in 2016 with a laparoscopic, antecolic Roux-en-Y gastric bypass. The patient did well for two to three weeks post-surgery but then developed new symptoms of cycles of debilitating left upper quadrant abdominal pain associated with abdominal distention, nausea and difficult bowel movements every four to five-days and relieved by eventual bowel movements. A computed tomography (CT) of the abdomen was performed which showed no clear evidence of internal hernia. Initially, her condition was managed medically with bowel regimen and diet modification but due to the persistence of her symptoms, she underwent diagnostic laparoscopy to rule out intermittently incarcerating and reducing internal hernia. At laparoscopic exploration, there was no evidence

Citation: Makarawo TP, Bastawrous S, Bastawrous A (2018) Splenic Flexure Mobilization for Subacute Large Bowel Obstruction following Gastric Bypass: A Case Report. Int J Surg Res Pract 5:067. doi.org/10.23937/2378-3397/1410067

Received: April 06, 2018: Accepted: April 30, 2018; Published: May 02, 2018

Copyright: (C) 2018 Makarawo TP, et al. This is an open-access article distributed under the terms of the Creative Commons Attribution License, which permits unrestricted use, distribution, and reproduction in any medium, provided the original author and source are credited. 


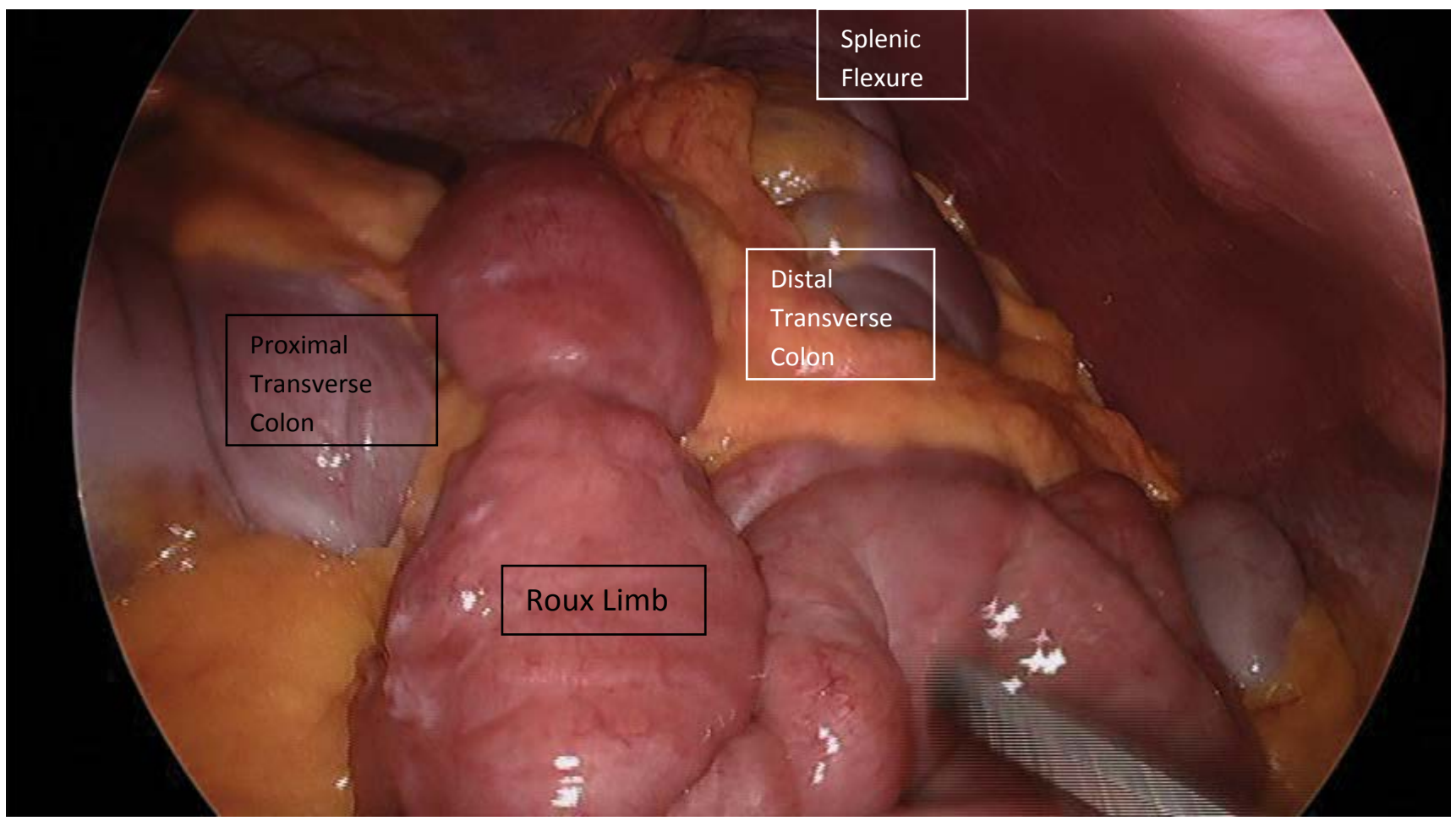

Figure 1: Intraoperative view demonstrating the antecolic Roux limb overriding the transverse colon heading towards an abnormally high splenic flexure.

of internal hernia but the ascending and transverse colon were markedly distended with dense adhesions between the transverse colon and omentum thought to be the source and so freed laparoscopically. However, postoperatively, the patient had persistent obstructive symptoms.

As a result, following colorectal surgery consultation, she underwent a second laparoscopic exploration procedure. At this exploration, it appeared that the Roux limb from the RYGB appeared to be impinging the distal transverse colon as there was a transition point seen where it was overriding the colon (Figure 1). In addition, the splenic flexure of the colon itself was abnormally high and densely adherent to the superior pole of the spleen causing an abnormal axial twist of the colon at this point. The descending colon beyond this point was decompressed. To correct the colonic obstruction, a laparoscopic splenic flexure mobilization was performed. The steps used were first releasing the abnormal adhesions of the colon to the upper pole of the spleen. This required tedious and careful dissection to avoid splenic injury. Once these dense adhesions were released, the standard steps of splenic flexure mobilization were followed with division of the normal anatomic attachments of the gastrocolic, splenocolic, pancreatico-colic and reno-colic ligaments. The descending colon was only partially mobilized by dividing the upper attachments at the white line of Toldt. Upon completion of the splenic flexure mobilization, the transverse colon was released into the lower abdomen, clearly free of impingement from the Roux limb, concluding the procedure.

The patient made an uneventful recovery with dis- charge on the day of surgery. At surgical follow-up, the patient reported complete resolution of her subacute colonic obstructive symptoms with regular bowel movements without the aid of laxatives and no abdominal pain. There has been no recurrence of the obstructive symptoms since then.

\section{Discussion}

Although bowel obstruction secondary to internal hernia is a well-documented complication of RYGB surgery, the optimum approach to minimize the risk of obstruction is controversial. A recent meta-analysis observed increased rates of small bowel obstruction and internal hernia with retrocolic, retrogastric positioning of the roux limb due to increased potential defects that may contribute as internal hernia sites [4]. The laparoscopic approach has been associated with increased small bowel obstructions compared to the open approach and this has been hypothesized to be due to the absence of adhesions that would normally stabilize the small bowel and prevent bowel obstruction within internal hernias [2].

Indeed, as was initially thought in our case, atypical small bowel obstruction features can occur when the small bowel intermittently becomes trapped then reduced at the site of the internal hernia [1] prompting laparoscopic exploration.

To our knowledge, this is therefore the first case report of large bowel obstruction following open or laparoscopic RYGB. CT scanning is not always diagnostic of bowel obstruction following RYGB, but in this case the significant ascending and transverse colon distention 
provided some useful clues to this unique cause of bowel obstruction.

Normally, the antecolic Roux limb does not compress the transverse colon enough to cause colonic obstruction. However, in this case, we believe the abnormally cranial splenic flexure elevated the distal transverse colon higher within the abdominal cavity limiting the amount of room between it and the antecolic Roux limb leading to impingement and thus mechanical bowel obstruction. Although we believe the abnormal attachments between the colon and the superior pole of the spleen are likely congenital, there may have been an iatrogenic component from her prior bariatric surgeries as well as evidenced by the dense adhesions encountered during the dissection.

Although one may argue that in this case, a retrocolic Roux limb may have eliminated the potential for colonic impingement, the abnormal anatomic orientation heavily contributing to this complication is rare enough to make it unnecessary to alter one's practice of antecolic Roux limb orientation if they so choose.

\section{Conclusion}

Considering the unusual presentation of mechanical large bowel obstruction following RYGB, splenic flexure mobilization provided an effective solution to relieve the obstruction in this unique situation.

\section{References}

1. Harakeh AB (2011) Complications of Laparoscopic Rouxen-Y Gastric Bypass. Surgical Clin N Am 91: 1225-1237.

2. Capella RF, lannace VA, Capella JF (2006) Bowel obstruction after Open and Laparoscopic Gastric Bypass Surgery for Morbid Obesity. JACS 203: 328-335.

3. Felscher J, Brodsky J, Brody F (2003) Small bowel obstruction after laparoscopic Roux-en-Y gastric bypass. Surgery 134: 501-505.

4. Harakeh AB, Kallies KJ, Bogert AJ, Kothari SN (2016) Bowel obstruction rates in antecolic/antegastric versus retrocolic/retrogastric Roux limb gastric bypass: A meta-analysis. Surg Obes Relat Dis 12: 194-198. 